Ann. Geophysicae 16, 743-753 (1998) @ EGS - Springer-Verlag 1998

\title{
Superposed epoch analysis applied to large-amplitude travelling convection vortices
}

\author{
H. Lühr ${ }^{1}$, M. Rother ${ }^{1}$, T. Iyemori ${ }^{2}$, T. L. Hansen ${ }^{3}$, R. P. Lepping ${ }^{4}$ \\ ${ }^{1}$ GeoForschungsZentrum Potsdam, Telegrafenberg, D-14473 Potsdam, Germany \\ ${ }^{2}$ Data Center for Geomagnetism and Space Magnetism, Kyoto University, Kyoto 606-01, Japan \\ ${ }^{3}$ Auroral Observatory, University of Troms $\varnothing$, N-9037 Troms $\varnothing$ Norway \\ ${ }^{4}$ NASA/Goddard Space Flight Center, Electrodynamics Branch, Greenbelt, MD 20771, USA
}

Received: 15 September 1997 / Revised: 9 January 1998 / Accepted: 22 January 1998

\begin{abstract}
For the six months from 1 October 1993 to 1 April 1994 the recordings of the IMAGE magnetometer network have been surveyed in a search for largeamplitude travelling convection vortices (TCVs). The restriction to large amplitudes $(>100 \mathrm{nT})$ was chosen to ensure a proper detection of evens also during times of high activity. Readings of all stations of the northern half of the IMAGE network were employed to check the consistency of the ground signature with the notation of a dual-vortex structure moving in an azimuthal direction. Applying these stringent selection criteria we detected a total of 19 clear TCV events. The statistical properties of our selection resemble the expected characteristics of large-amplitude TCVs. New and unexpected results emerged from the superposed epoch analysis. TCVs tend to form during quiet intervals embedded in moderately active periods. The occurrence of events is not randomly distributed but rather shows a clustering around a few days. These clusters recur once or twice every 27 days. Within a storm cycle they show up five to seven days after the commencement. With regard to solar wind conditions, we see the events occurring in the middle of the IMF sector structure. Large-amplitude TCVs seem to require certain conditions to make solar wind transients 'geoeffective', which have the tendency to recur with the solar rotation period.
\end{abstract}

Key words. Ionosphere (Aural ionosphere; Ionospheremagnetosphere interactions) - Magnetospheric Physics (current system)

\section{Introduction}

The polar cusp/cleft region which maps the magnetopause and adjacent layers is rich in transient events in

Correspondence to: H. Lühr the magnetic recordings. Kleymenova et al. (1985) found that these irregular pulsations exhibiting mean periods around $8 \mathrm{~min}$ occur for both interplanetary magnetic field (IMF) polarities, $B_{z}>0$ and $B_{z}<0$, and that they show a clear occurrence peak in the pre-noon sector between 06 and 11 magnetic local time (MLT).

The early studies of the magnetic impulse events (MIE) were part of a search for ground signatures of sporadic reconnection at the magnetopause termed flux transfer event (FTE) (e.g. Goertz et al., 1985; Lanzerotti et al., 1986, 1987). The motivation for these studies came from theoretical works predicting the ground signature of FTEs (Saunders et al., 1984; Southwood, 1987; Lee, 1986).

An alternative interpretation was offered by FriisChristensen et al. (1988) who related their observed MIEs to transient changes in solar wind dynamic pressure and IMF orientation. By using an array of magnetometers they showed that the magnetic deflections matched an interpretation of steady ionospheric current systems moving at a velocity of several $\mathrm{km} / \mathrm{s}$ from east to west overhead the stations. This class of magnetic impulses was termed travelling convection vortices (TCV). From an extensive statistical study Glaßmeier et al. (1989) could deduce the characteristic features of TCV events. Basic elements are Hall current vortices with alternating senses of rotation which form around field-aligned current (FAC) filaments. When moving overhead a ground station at a velocity of several $\mathrm{km} / \mathrm{s}$ quasi-sinusoidal magnetic signatures are produced which are in quadrature at the two components, $\mathrm{H}$ and $\mathrm{D}$. The occurrence frequency versus local time, as reported by these authors, showed a clear peak at 08 MLT.

In several later studies details of the TCVs were further refined (e.g. Sibeck, 1990; Glaßmeier, 1992; Lühr and Blawert, 1994; Lühr et al., 1996). For several cases a direct link between TCVs and solar wind dynamic pressure variations could be established, but to date it was impossible to clarify whether this is the only or, at least, the primary cause of TCVs. If caused by a solar 
wind pressure front, TCVs should show up equally frequent on the pre- and post-noon side. There are a few reports on TCVs from the afternoon side in the literature (e.g. Vo et al., 1994; Lühr and Blawert, 1994), but the observed amplitudes were much smaller than those in the pre-noon sector. Sitar et al. (1997) reported even more puzzling results. When surveying solar wind data for transient dynamic pressure changes and studying the associated ground magnetic responses, they found that a number of different kinds of ground responses occurred but many of them are neither consistent with theoretical nor observational models. Their conclusion is that the ground responses result from a more complicated combination of internal and external controls. A question that could be asked is: do the magnetospheric conditions for propagating the signals from the magnetopause to the polar ionosphere differ from sector to sector in local time and/or do they depend on external factors like the solar wind conditions?

In this study we present a statistical survey of largeamplitude TCV events from the recordings of the IMAGE magnetometer network during the six months, October 1993 through March 1994. The purpose of the study is to find out the conditions that favour the generation of large and clean TCV events. Unfortunately, the coverage of IMF data is not very good. Either the IMP-8 spacecraft was far out at the flanks $\left(\left|\mathrm{Y}_{\mathrm{GSE}}\right|>20 \mathrm{R}_{\mathrm{E}}\right)$, in the tail, or there are data gaps. A reason for this is that IMP-8 was rarely tracked from the European sector. To overcome this problem at least partly, we have taken a number of magnetospheric activity indices into account which can be regarded as a proxi for IMF conditions.

In a recent study Crooker and McAllister (1997) have covered the same time period (winter 1993/94) searching for solar and solar wind features in association with recurrent magnetic storms. We will make use of their results to see how our TCVs are related to magnetic storms and/or solar wind conditions.

For the identification of the TCV events we employed recordings of the IMAGE magnetometer network. Since TCVs are a high-latitude phenomena, we have limited our search to stations of the northern half of the network. A list of the stations considered in this study is given in Table 1. The instruments sensing the magnetic variations are fluxgate magnetometers. Digital readings averaged over $10 \mathrm{~s}$ and with a resolution of better than 1 $\mathrm{nT}$ are stored. For a more detailed description of the network see Lühr (1994) and Lühr et al. (1998).

\section{Statistical analyses}

For the statistical survey data from the six months, October 1993-March 1994 of the extended IMAGE magnetometer network have been searched for largeamplitude TCVs. Only large TCVs have been considered, since they can easily be identified. Previous statistical studies have often been criticised of being biased toward quiet times, because TCVs occurring during active periods may have been masked by the background variations. Furthermore we wanted to establish the prevailing magnetospheric conditions which favour the growth of TCVs.

Events have been selected by visual inspection of latitudinally stacked plots. The selection criteria were:

1. Peak amplitude $>100 \mathrm{nT}$

2. Amplitude maximum in $\mathrm{Y}$ occurs at same latitude as minimum in $\mathrm{X}$

3. $\mathrm{X}$ is in anti-phase north and south of the minimum

4. Features propagate in east-west direction at several $\mathrm{km} / \mathrm{s}$

These criteria in essence are identical with the features listed by Glaßmeier et al. (1989) to characterise TCVs.

Figure 1 shows as an example a $2 \mathrm{~h}$ period which contains two large-amplitude TCV events. The central latitude at which the $\mathrm{X}$ (northward) component switches sign is near BJN (71.2 $2^{\circ}$ magnetic latitude). The amplitudes of the peaks in Y (eastward) at this site range around $300 \mathrm{nT}$. The magnetic local time (MLT) of occurrence of these events is 0930 and 1030 MLT. (Note that MLT at the Svalbard stations is about $3 \mathrm{~h}$ ahead of UT. The declination at these stations amounts only to a few degrees. Therefore, it makes little difference whether geomagnetic $(\mathrm{H}, \mathrm{D})$ or geographic $(\mathrm{X}, \mathrm{Y})$ components are used.)

The propagation velocity of the features has been estimated by determining the phase delay of the $\mathrm{Y}$ components between BJN and JAN. (Recordings of the station JAN are plotted as the bottom trace in Fig. 1.) The signals appear delayed at JAN by 60 and $70 \mathrm{~s}$ for
Table 1. Locations of the magnetometer stations IMAGE (International Monitor for Auroral Geomagnetic Effects)

\begin{tabular}{|c|c|c|c|c|c|c|}
\hline \multirow[t]{2}{*}{ Station name } & \multirow[t]{2}{*}{ Code } & \multicolumn{2}{|c|}{ Geographic } & \multicolumn{2}{|c|}{ AACGM magnetic ${ }^{a}$} & \multirow{2}{*}{$\begin{array}{l}\text { L-value } \\
10 \text { MLT }\end{array}$} \\
\hline & & Latitude & Longitude & Latitude & Longitude & \\
\hline Ny Ålesund & NAL & 78.92 & 11.95 & 75.9 & 112.7 & - \\
\hline Longyearbyen & LYR & 78.20 & 15.82 & 75.0 & 113.4 & - \\
\hline Hornsund & HOR & 77.00 & 15.60 & 73.9 & 110.8 & 10.6 \\
\hline Hopen Island & HOP & 76.51 & 25.01 & 72.7 & 116.2 & 9.9 \\
\hline Bear Island & $\mathrm{BJN}$ & 74.50 & 19.20 & 71.2 & 109.2 & 9.1 \\
\hline Tromsø & TRO & 69.66 & 18.94 & 66.3 & 103.6 & 6.4 \\
\hline Jan Mayen & JAN & 70.90 & 351.30 & 70.2 & 84.6 & 8.8 \\
\hline
\end{tabular}

${ }^{a}$ Altitude Adjusted Corrected Geomagnetic coordinates (Baker and Wing, 1989) 
I M A G E MAGNETOMETER 1994-01-18

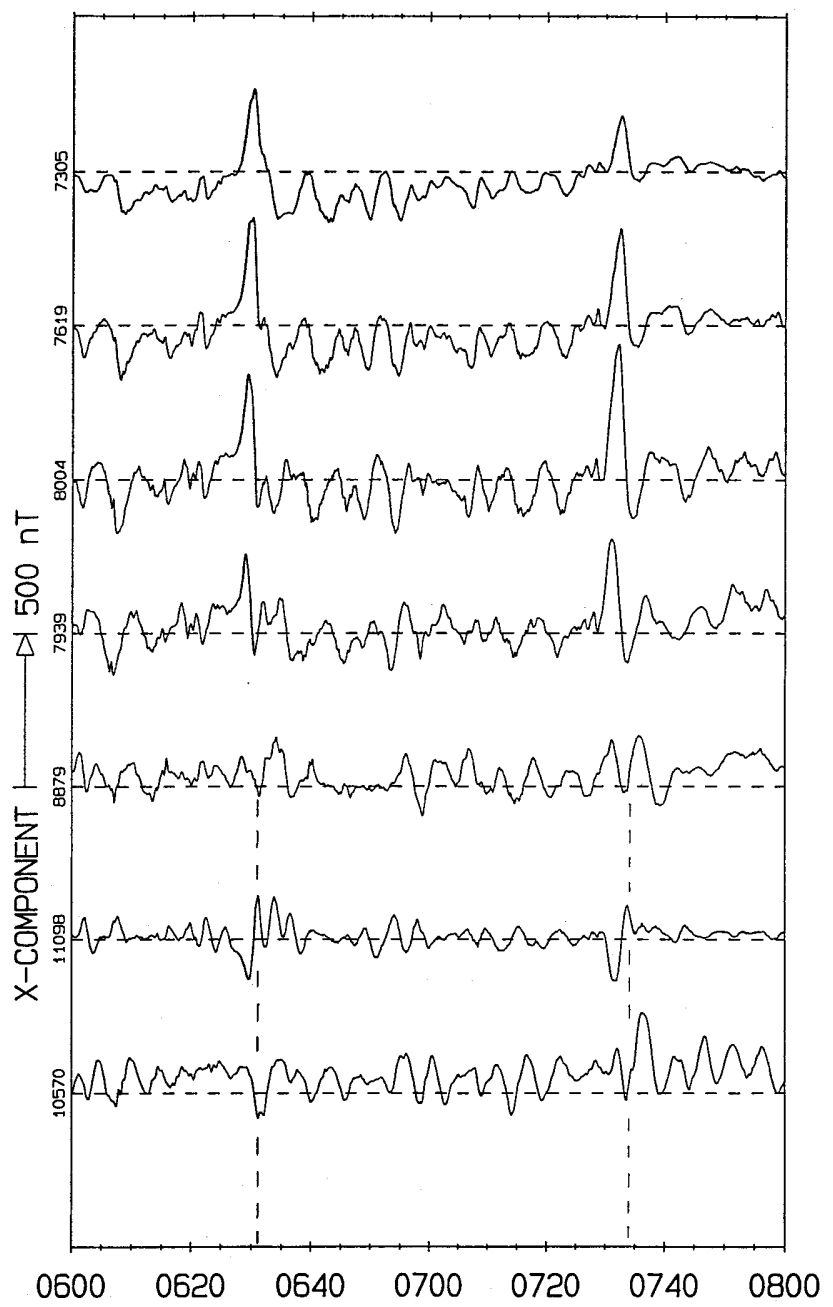

Fig. 1. Magnetograms obtained along a meridian chain from Svalbard to Scandinavia showing as an example the occurrence of two large-amplitude TCVs (dashed lines). The bottom trace shows the

the first and second event, respectively. Taking into account the azimuthal separation of these stations $\left(24.6^{\circ}\right.$ or $872 \mathrm{~km}$, see Table 1) westward propagation speeds of $14.5 \mathrm{~km} / \mathrm{s}\left(0.41^{\circ} / \mathrm{s}\right)$ and $12.5 \mathrm{~km} / \mathrm{s}\left(0.35^{\circ} / \mathrm{s}\right)$ result. From the description of the presented example we see that the two TCV events fulfil our selection criteria.

In our survey of six months of data we found 19 large-amplitude TCV events. The dates and times of the events are listed in Table 2. These events are not evenly distributed in time. As can be seen from the top graph of Fig. 2, TCVs occurred much more frequently in the first half of the six months period than in the second. In addition the separation between adjacent events does not follow a normal distribution. The second graph of Fig. 2 shows that it is most likely to find TCVs separated by less than 5 days. The next highest bin is 10 to 15 days and less significant is the bin 20 to 25 days. TCVs seem to cluster in time and these clusters tend to repeat after half a solar rotation or a full rotation. It should be noted here that days with more than one event have only been counted once in the two upper graphs of Fig. 2. If these
I M A G E MAGNETOMETER 1994-01-18

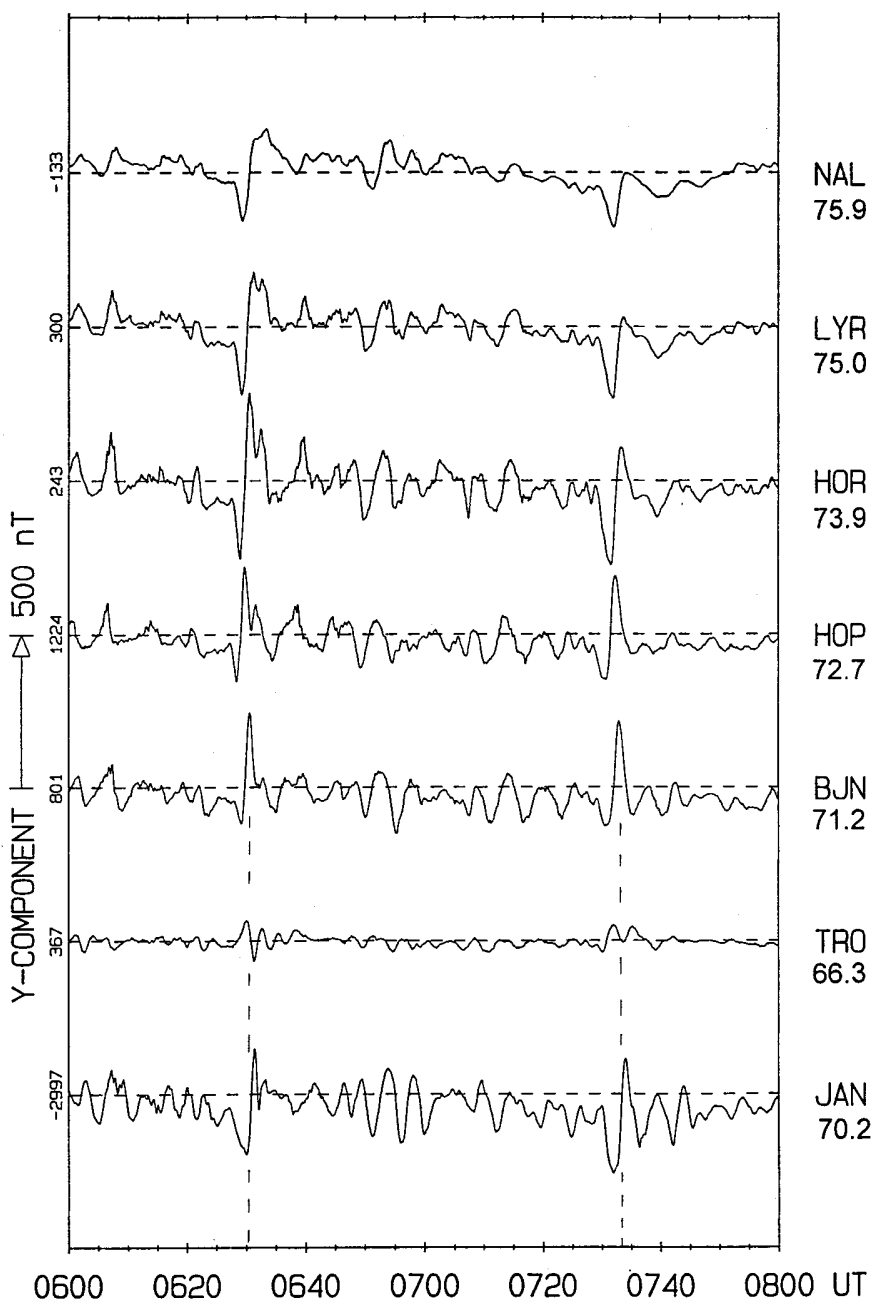

recording from the station Jan Mayen, $24^{\circ}$ further west. Corrected magnetic latitudes are given below the name codes

additional events are also taken into account, the tendency towards clustering becomes even more evident.

The local time of occurrence shown at the bottom of Fig. 2 is limited to the $2 \mathrm{~h}$ around 10 MLT. This finding accords well with earlier studies (e.g. Sibeck, 1990; Lühr and Blawert, 1994) that report largest amplitudes of TCVs in the pre-noon sector.

For 15 out of 19 events the propagation velocity could be estimated from phase delays of the $\mathrm{Y}$ signature between BJN and JAN; for the remaining cases there are no JAN data available see Table 2). In all these 15 cases we found a westward drift at an average speed of $v=10.5 \pm 3.2 \mathrm{~km} / \mathrm{s}$. This value is at the high end of reported velocities but is not out of range. Possible explanations for the elevated value will be discussed later.

Interplanetary magnetic field (IMF) data obtained by the IMP-8 spacecraft are available for only 6 out of 19 events (see Table 3 ). In spite of this small number and even with the unfavourable position of IMP-8, as outlined, some systematic field configurations seem to 
Table 2. List of large-amplitude travelling convection vortex events

\begin{tabular}{lll}
\hline Date & $\begin{array}{l}\text { Key times of TCVs } \\
\text { Bear Island }\end{array}$ & $\begin{array}{l}\text { (peak in Y comp.) } \\
\text { Jan Mayen }\end{array}$ \\
\hline $93-10-12$ & $0635: 40$ & - \\
$93-10-13$ & $0905: 10$ & - \\
$93-10-24$ & $0743: 30$ & $0744: 50$ \\
$93-10-28$ & $0748: 50$ & $0751: 40$ \\
$93-11-08$ & $0618: 40$ & $0619: 50$ \\
$93-11-20$ & $0721: 30$ & $0723: 20$ \\
$93-11-23$ & $0729: 00$ & $0731: 30$ \\
$93-11-26$ & $0716: 30$ & $0717: 30$ \\
$93-12-06$ & $0619: 30$ & - \\
$93-12-07$ & $0636: 50$ & - \\
$93-12-21$ & $0612: 00$ & $0613: 50$ \\
$93-12-21$ & $0727: 40$ & $0728: 50$ \\
$93-12-22$ & $0655: 00$ & $0656: 30$ \\
$94-01-16$ & $0509: 40$ & $0510: 20$ \\
$94-01-18$ & $0630: 20$ & $0631: 20$ \\
$94-01-18$ & $0732: 50$ & $0734: 00$ \\
$94-02-02$ & $0804: 00$ & $0805: 50$ \\
$94-02-21$ & $0726: 20$ & $0728: 40$ \\
$94-03-14$ & $0637: 40$ & $0639: 20$ \\
\hline
\end{tabular}
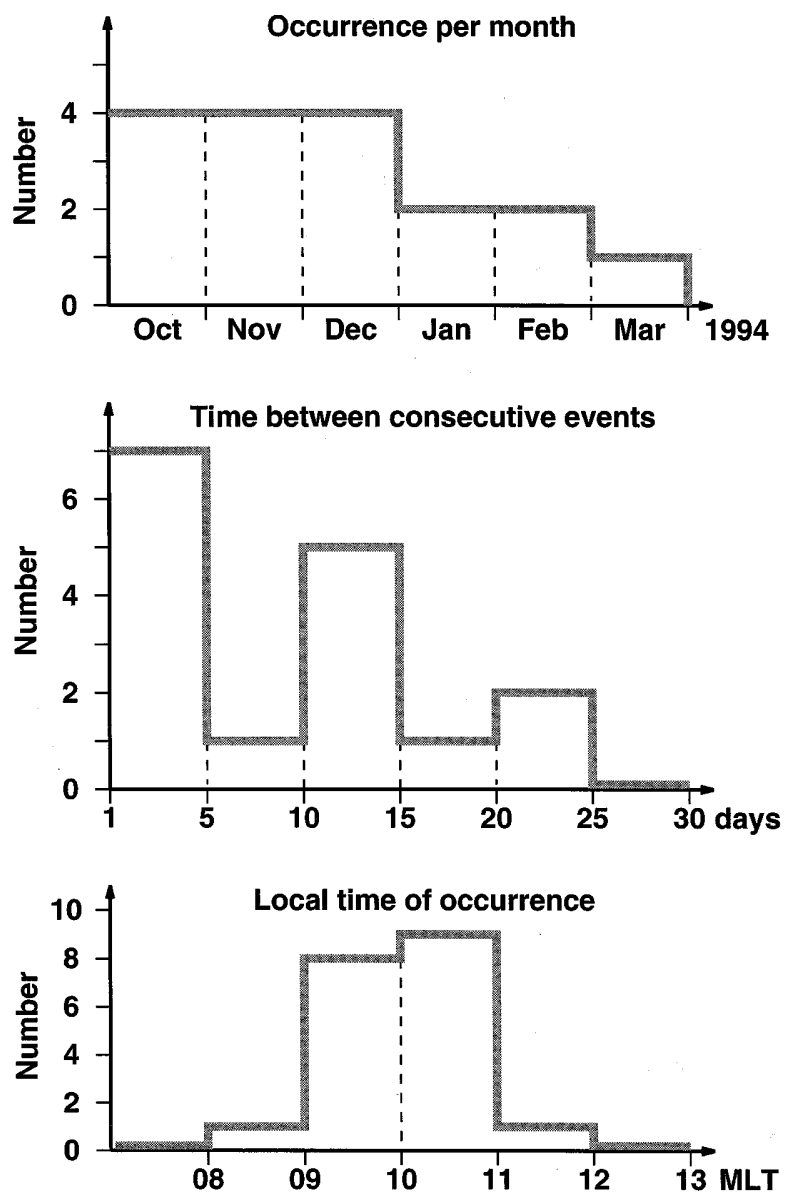

Fig. 2. Occurrence statistic of the 19 selected events. (Top) There is a clearly higher occurrence rate during the first 3 months than during the later 3. (Middle) The occurrence probability is not consistent with a random distribution. Groups of TCVs tend to recur after 13 or 27 days. Multiple occurrences per day have not been taken into account. (Bottom) The local time of occurrence is limited to $2 \mathrm{~h}$ in the pre-noon sector emerge. During 5 out of 6 events IMP-8 was in the toward sector (IMF pointing towards the sun) and $B_{Y}$ being negative or zero. For the $B_{z}$ component in geocentric solar magnetic (GSM) coordinates we find positive values for three cases, one around zero and in two events there is a minus/plus sign change when taking a 5 to $10 \mathrm{~min}$ propagation delay from IMP-8 to ionosphere into account. The field strength exhibited quite normal values between 5 and $7 \mathrm{nT}$. These results seem to suggest a relation between TCV occurrence and the large-scale solar wind sector structure. A more detailed discussion of this aspect will be given in Sect. 4.3.

\section{Superposed epoch analyses}

Our finding of the previous section that TCVs tend to cluster and that the clusters show a recurrent period of 13 days suggests that large-amplitude TCVs are linked in some way to the solar rotation and/or the magnetic activity. To find out whether there are particular conditions prevailing before or during the appearance of our TCVs we applied a superposed epoch analysis to several data sets.

Figure 3 shows a superposition of the magnetic field traces at BJN of all 19 events. The intervals comprise $7 \mathrm{~h}$ before and $5 \mathrm{~h}$ after the TCV. As the key time the peak of the $\mathrm{Y}$ component at $\mathrm{BJN}$, the station which was in most cases the closest to the vortex centres, has been used. For the time axis we used the event time (ET). On this time scale $0700 \mathrm{ET}$ is the key time of all events. As can be deduced from a comparison with the bottom graph of Fig 2, the key time, $07 \mathrm{ET}$, corresponds on average to 10 MLT.

From the three magnetic field traces in Fig 3 we can read the average conditions accompanying our events. This is possible, since quite night-time values have been subtracted from the readings. The base lines of the stations used are checked regularly and found to be reliably stable (Lühr et al., 1998). The northward, X, component is negative during the morning hours, it goes through zero and becomes positive in the afternoon. This implies a location of BJN under the westward electrojet during the morning hours and under the eastward jet in the afternoon. The average level of Y can be interpreted as the deviation of the electrojet orientation from the geographic east-west direction. Finally, the $\mathrm{Z}$ component varying more or less in anti-phase with $\mathrm{X}$ suggests that $\mathrm{BJN}$ is generally located to the north of the electrojet centres.

The TCVs, clearly outstanding in the Y component, occur at a time when all three components are close to zero, i.e. there is no electrojet activity in the vicinity of the station. On top of the average traces we have plotted, as vertical bars, the standard deviations computed from the whole stack of superposed traces. Smallest deviations are found in the vicinity of the events. Thus, we may keep in mind the fact that the absolute levels of magnetic deflections are small and also that the variability shows a minimum around our TCVs. 
Table 3. IMP-8 data of interplanetary magnetic field

\begin{tabular}{|c|c|c|c|c|c|c|c|c|}
\hline Date & Time & \multicolumn{4}{|c|}{ Interplanetary magnetic field } & \multicolumn{3}{|c|}{ Position in $\mathrm{R}_{\mathrm{E}}(\mathrm{GSM})$} \\
\hline $93-10-28$ & 0730 & Toward & - & + & 5 & 13 & -27 & 12 \\
\hline $93-11-20$ & 0710 & Toward & - & $-\rightarrow+$ & 5 & -13 & -26 & 15 \\
\hline $93-12-22$ & 0640 & Toward & - & $-\rightarrow+$ & 7 & 2 & 35 & 11 \\
\hline $94-01-16$ & 0500 & Toward & - & $\approx 0$ & 6 & 10 & 29 & 22 \\
\hline
\end{tabular}

The right panel of Fig 3 shows an enlargement of the variations around the key time. From them we may get the average properties of our events. The Y component shows a large positive peak which is expected at the centre between the two current vortices. Its amplitude is about $200 \mathrm{nT}$ and the half-value width amounts to 2 min. In $X$ we have a much smaller bipolar variation which is consistent with the passage of a twin-vortex system close to their centres. The $\mathrm{Z}$ component repeats

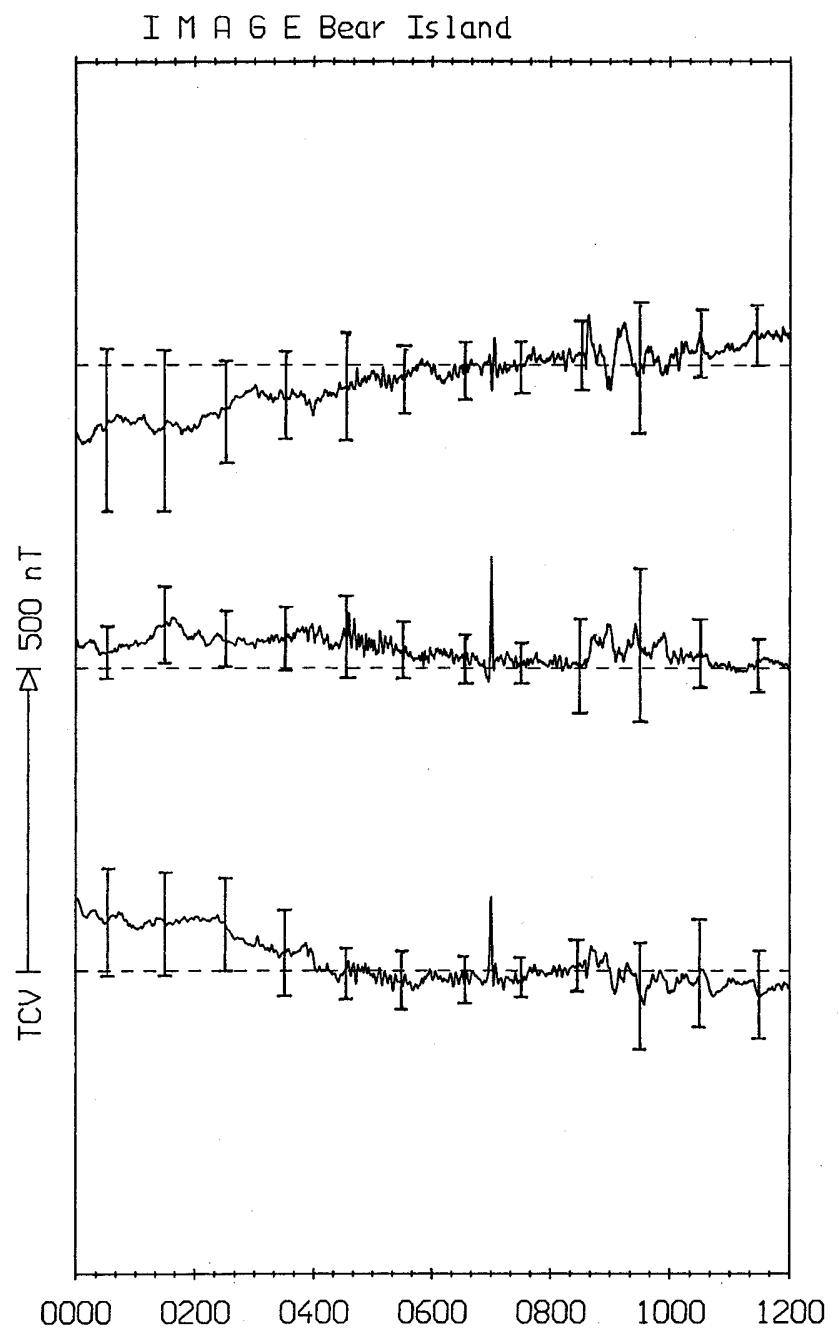

Fig. 3. Average magnetogram obtained by superposing the traces of the 19 events at Bear Island. The time scale is given in event time (ET) where the key time (peak of Y comp.) is set to $07 \mathrm{ET}$. The vertical bars primarily the variations in $\mathrm{Y}$, which is, to a large extent due the induction effect in the ground and the surrounding ocean.

The conditions found by superposing the magnetic field traces of a single station reflect the local state of activity and could be interpreted as local time effects. To get an idea of the global state of activity we conducted a superposed epoch analysis with the $K_{p}$-index of the 17 days when events were detected. Figure 4 shows the

I MAGE Bear Island

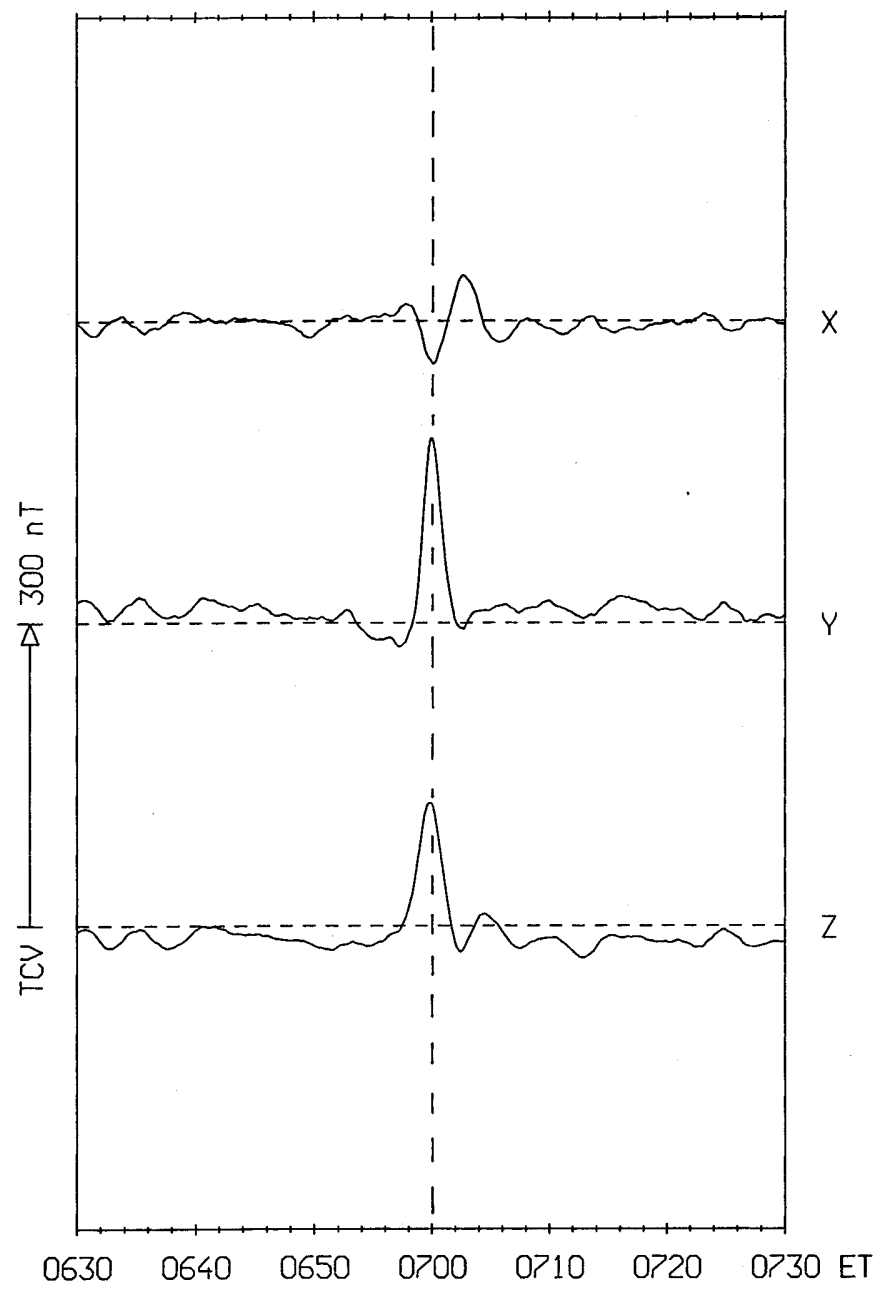

represent the standard deviation computed from the whole stack of superposed traces. In the vicinity of the TCV the deviations are smallest 


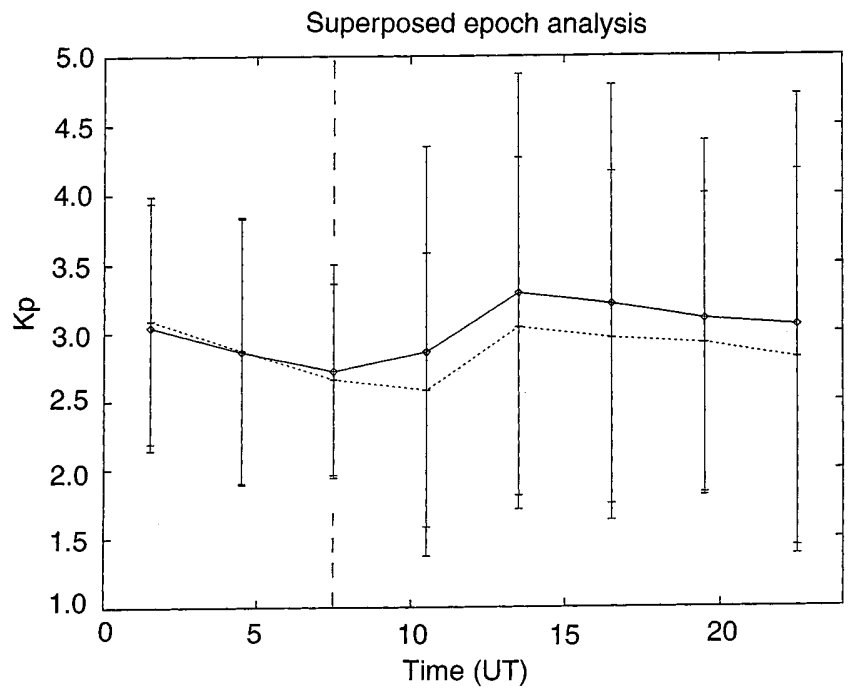

Fig. 4. Superposed epoch analysis of the $K p$ index. The solid line is for all 19 events, The dashed line is minus 21, February 1994 where a major storm commenced at 0901 UT. Vertical bars indicate the standard deviation. TCVs occur near a local activity minimum again accompanied by smallest deviations

results of the $K_{p}$ superposition. Since our event on 21, February 1994 was followed by a big magnetic storm, Fig. 4 contains two traces (solid line: all days included, dashed line: minus 21, February). The two lines match almost perfectly before the storm (SSC: 0901 UT), after the onset the solid line is systematically higher, as expected. In spite of this we may note that $K_{p}$ continuously decreased during the $7 \mathrm{~h}$ before the event from about 3 to a minimum of about 2.6, around the time of the event. Afterward, both traces, with and without the 21, February storm, increased again. The standard deviations are indicated again by vertical bars. As a resume we may say that our TCVs occur during relative minima in $K_{p}$ ranging around $3-$ and the variability from event to event is relatively small.

The apparent tendency of TCV clusters to recur with a period of about 13 or 27 days suggests that there may be a relation to magnetic storms. As an indicator for the storm activity we have used the Dst index for a superposed epoch analysis. Figure 5 shows the results of the analysis. The superposed Dst index is displayed for the interval 10 days before the event up to 5 days after. The standard variations resulting from this analysis are quite large therefore the interpretation of individual features requires some care. In spite of this uncertainty a general trend can be read from the curve. Eight days before the event the average $D s t$ value had its maximum, close to zero. Thereafter it gradually decreased, reaching the minimum four days later. During the subsequent days it gradually increased again until the event time. Later $D s t$ varies around $-20 \mathrm{nT}$.

Prominent features in Fig. 5 are steep negative slopes during the days $-7,-6$, and -4 . These ramps can be associated with storm commencements. Since TCVs tend to cluster around consecutive days, one commencement can produce several ramps at an increment of whole days. After day -4 the storm activity decreases as

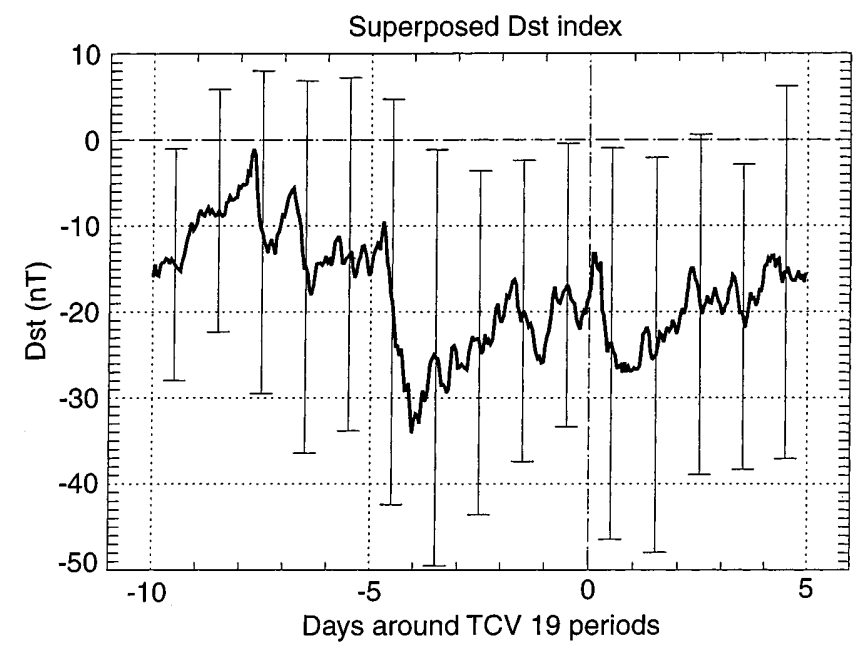

Fig. 5. Superposition of the Dst index for the 10 days before and 5 days after the TCV events. In spite of the large variability it is apparent that the events occur during a local maximum some 5 days after a storm commencement

implied by the rising $D s t$ level, reaching a relative maximum just after the key time. Looking at the standard deviation we again find that the variations just before the key time are smaller than earlier or later.

In summary: large-amplitude TCVs tend to occur about a week after the commencement of a magnetic storm during interleaved periods of relative quietness.

After having investigated the relationship with magnetic storms it would be interesting to look into the dependence on substorms. An index describing the substorm activity rather reliably is AE. Unfortunately $\mathrm{AE}$ indices are still not available for the period under study. As a proxy for the auroral activity the midlatitude geomagnetic index "ASY" published by the Data Analysis Center for Geomagnetism and Space Magnetism, Kyoto University can be used (Clauer et al., 1983). The "ASY" index represents the range of deviations of mid-latitude stations after subtracting $S_{q}$ and Dst variations (Iyemori et al., 1994).

In Fig. 6, top panel, a superposition of the ASY components is shown. Here again the $7 \mathrm{~h}$ before and $5 \mathrm{~h}$ after the event are plotted. The range between the two traces, ASY-D top and ASY-H bottom, can be regarded as a measure for the auroral activity. To date no reliable conversion factor between ASY and AE has been published, but as a qualitative statement we may note that the range minimises just before the occurrence of the TCV. This means that all the substormrelated deflections caused e.g. by the partial ring current, cross-tail current and current wedge FACs are reduced.

For completeness we also plotted the superposition of the two components of the index "SYM". The component SYM-H is quasi identical with the Dst, ring storm, index, except that we have a 1 min resolution rather than the $1 \mathrm{~h}$ of the latter. Here again we find a positive slope as in Fig. 5 implying a closed magnetosphere. It has been suggested by several authors that TCVs are associated with pressure pulses in the solar 


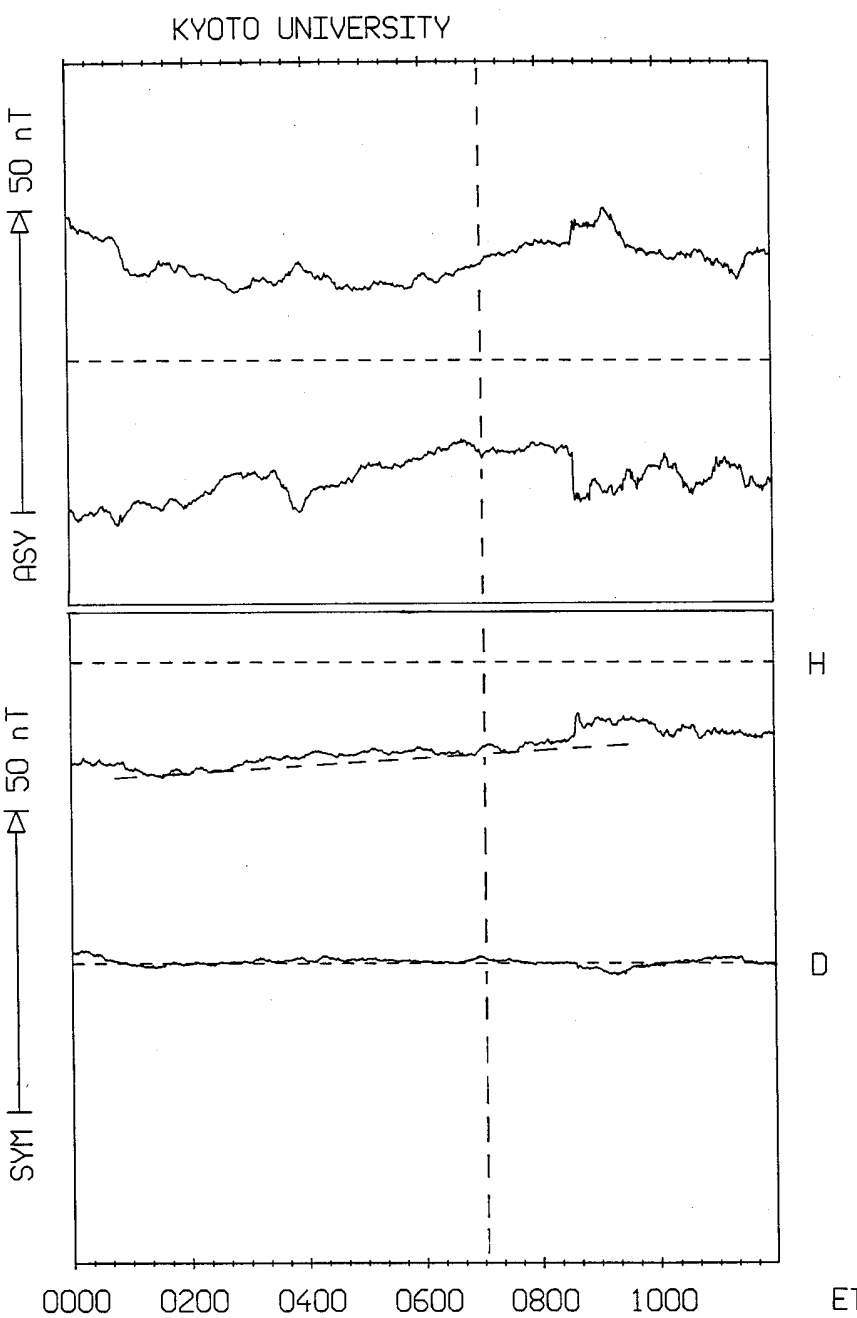

Fig. 6. Superposition of the ASY and SYM indices. The key time of the events $(07 \mathrm{ET})$ is marked by a vertical line. The range between the ASY traces can be interpreted as a proxi for the auroral activity. It minimises near the key time. The SYM-H component is an equivalent to the Dst index. It rises steadily during the hours before the key time. Note that there is no signature of an SI-type compression

wind (e.g. Friis-Christensen et al., 1988; Sibeck, 1990; Lühr et al., 1993). The SYM-H component would be appropriate to identify sudden commencements (SC) or sudden impulses (SI). There is, however, neither in SYM-H nor in SYM-D a signal that could be accounted for as a global compression of the magnetosphere at the time of the events.

In previous studies on TCVs their cause has primarily been related to transients in the solar wind. This analysis, however, reveals that the appearance of largeamplitude TCVs depends also on certain activity conditions in the magnetosphere.

\section{Discussion}

In this section we want to recall the results obtained in our statistical study and discuss their implications in the context of earlier published characteristics of TCVs.

\subsection{Statistical properties of TCVS}

Our selection criteria are designed for the identification of ionospheric current vortices travelling in east/west direction overhead a meridional chain of magnetometer stations. The underlying magnetospheric/ionospheric current system comprises a pair of anti-parallel fieldaligned current (FAC) filaments. In the ionosphere the current continuity is maintained by Pedersen currents. As a secondary effect Hall currents are generated which circulate around the centres of the FAC filaments. Magnetic field variations observed on the ground are predominantly caused by these Hall current vortices. As an example for a model of the proposed current system see Lühr and Blawert (1994).

All our events showed a dominant positive deflection of the $\mathrm{Y}$ component in the centre of the system although the sign of the $\mathrm{Y}$ deflection has not been a selection criterion. The positive peak in $\mathrm{Y}$ implies a sequence of FACs directed downward in the west and upward in the east. This kind of polarity has been identified in many studies as being associated with prominent TCV signatures.

TCV events are known to go through a considerable development during their lifetime. On their way from the initiation near noon to the flanks TCV signatures first grow in amplitude and later decay (e.g. Lühr and Blawert, 1994; Moretto et al., 1997). These authors have shown that the part of a TCV associated with the sequence of FACs first downward then upward (as studies here) goes through its maximum epoch around the 10 MLT time sector. This is fully consistent with out findings that all large-amplitude TCVs were detected in the two hours around 10 MLT.

During our six month survey we detected 19 events. When considering that we only had two hours in the favourable time sector, we could have expected 12 times more events with a dense network covering all local times. The resulting number of 228 corresponds to more than one large-amplitude TCV per day on average. However, our statistic does not support a random occurrence of TCVs but rather a clustering around 3-5 days twice a month. Over these days TCVs seem to be an important phenomena for transferring energy from the day-side magnetopause to the high-latitude ionosphere.

The propagation direction of all events was westward. This is also in accord with the widely accepted concept assuming a source region at the day-side magnetopause, which is propagated tailward by the solar wind. The travel speed we deduced, about $10 \mathrm{~km} / \mathrm{s}$, is not completely out of range but rather high compared to velocities publish for other TCV events, which ranged around $5 \mathrm{~km} / \mathrm{s}$. The question we have to answer is, are large-amplitude TCVs faster than others, or are there some systematic configurations that make our TCVs look faster? We favour the latter explanation.

For a proper determination of the propagation velocity at least two meridian chains of magnetometers, appropriately spaced in longitude, would be required. We determined the velocity from a chain of stations and 
a single station. Proper results could be obtained, if the vortex's track was parallel to the line connecting the remote station with one of the stations on the chain. In our actual cases we don not precisely know the orientation of the velocity vector. Furthermore we know that the current vortices are not circular but elliptical in shape with their major axis skewed and the equatorward edge of the TCV leading the poleward. Lühr et al. (1996), from example, reported for their event a tilt angle of about $40^{\circ}$ with respect to geographic north.

We estimated the propagation speed from the phase delay between the $\mathrm{Y}$ components of the stations BJN and JAN. If we take the $40^{\circ}$ tilt angle and consider the latitudes of the two stations (see Table 1), we obtain average velocities of 8.8 and $6.2 \mathrm{~km} / \mathrm{s}$ for travel passes toward magnetic and geographic west, respectively, which is closer to expected values. Estimating the velocity from a two-point measurement assumes a steady motion of a static structure. Studies of TCVs on a global scale revealed (Moretto et al., 1997) that the individual vortices go through a considerable temporal development both in intensity and scale size during their life-time and that the propagation is far from a steady motion. Our velocity estimates should thus only be regarded as a first order estimate.

\subsection{Relation to large-scale convection}

Up to now we have limited our discussion to the local effects associated with TCVs. Is it possible from our study to determine the region within the global convection pattern where TCVs reach their largest amplitude? An answer to this question may be found when looking at Fig. 3. From averaged magnetic field traces of the station BJN close to the centre of the vortices we can see that TCVs appear at a time when all three magnetic field components are close to zero. This implies that largeamplitude TCVs are connected to the convection reversal region.

Figure 7 shows statistical patterns of high-latitude convection obtained by HF radar (Rouhoniemi and
Greenwald, 1996). The observations are from times of moderate geomagnetic activity levels $(2-<K p<3+)$ and are sorted by IMF angle. These are the conditions which were prevailing during our events: moderate disturbance level with IMF $B_{z}$ values been zero or positive. A TCV generated near noon and propagating westward will eventually pass the convection throat. We selected our events from the peak value in the magnetic field Y component which corresponds to a poleward convection driven by the two oppositely rotating vortices. Maximum amplitudes of these bursts were found around $10 \mathrm{MLT}$ at the station BJN $\left(71.2^{\circ}\right.$ magnetic latitude). This position fits nicely the poleward streaming region at the convection throat in the whole pattern of Fig. 7. It might be the favourable superposition of TCV-induced flows and large-scale convection that causes the confinement of the large amplitudes to this sector.

Other studies have reported more than two vortices, both on the pre- and post-noon side (e.g. Lühr and Blawert, 1994; Vo et al., 1994). It is, however, only one vortex pair (counter-clockwise/clockwise) on the morning side which could experience, on its way to the flank, the constructive superposition of flows at the convection throat. This fact might well account for the observation that all the other vortices exhibit much smaller ground signatures and thus were rejected by our selection criteria. To test this hypothesis it would be recommendable to include the large-scale convection in TCV studies for a series of events, e.g. by applying the AMIE (Assimilative Mapping of Ionospheric Electrodynamics) technique.

\subsection{Relation of magnetic activity}

A rather unexpected result of this study is the relation of the occurrence probability of large-amplitude TCVs to magnetospheric activity levels. It is widely accepted that TCVs are caused by transient disturbances in the solar wind such as pressure pulses and/or orientation changes. If we accept this view, we should try to find the

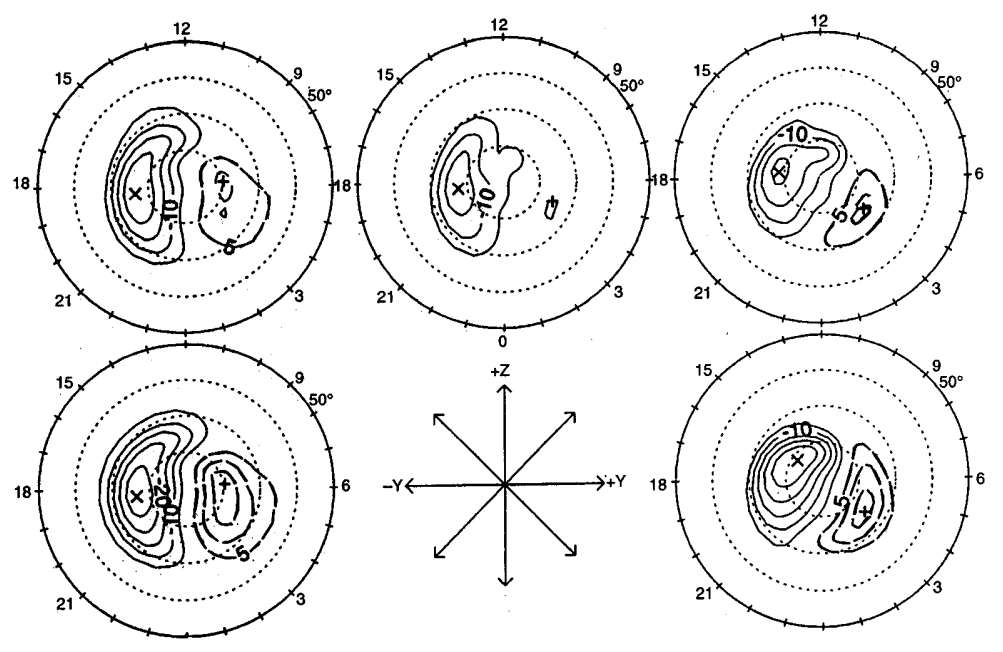

Fig. 7. Convection patterns sorted by IMF cone angle for a moderate geomagnetic disturbance level $(2-<K p<3+$ ) (from Rouhoniemi and Greenwald, 1996). For all conditions a convection throat appears near the 10 o'clock local time sector 
magnetospheric conditions which provide the necessary 'geoeffectiveness' to convert the solar wind transients into large-amplitude ionospheric disturbances.

From the superposition of the Dst index (see Fig. 5) we found TCVs to occur during the declining phase of a storm, about a week after the onset. The $K p$ values for the events ranged from 2.0 to 3.5. None showed up during totally quiet times and none during very active. Favourable conditions for the development of largeamplitude TCVs seem to exist during short quiet intervals (on the order of hours) embedded in moderately disturbed periods. The observed preference of positive IMF $B_{z}$ deduced from the sparse IMP-8 data and the rising $D s t$ value around the key time (see Figs. 5 and 6) both suggest that a closed magnetosphere enhances the 'geoeffectiveness'. We think that favourable conditions require a ring current filled to a certain degree. In more general terms, stable but appreciably steep plasma gradients are needed in the magnetosphere to support the transfer of the signal from the magnetopause to the ionosphere.

From Fig. 2 it is obvious that TCVs tend to occur on consecutive days. This tendency becomes even more clear when multiple occurrences per day are also considered. Figure 8 shows, as an example, the magnetogram from Bear Island for a $10 \mathrm{~h}$ interval centred at 07 UT, the time most favourable for detecting TCVs at this site. There are several solitary spikes in the Y component marking the occurrence of TCVs. The two largest have been included in our statistics. With a somewhat less stringent criteria we easily could have found five more events on this day. (Note the two negative spikes just before 04 UT which imply an opposite sequence of vortices, in accordance with the model of Lühr and Blawert (1994) for this early time sector.) The contrast of high frequency of occurrence during groups of 3 to 5 days to periods totally void of events makes it hard to discard the necessity of magnetospheric conditions favouring the formation of TCVs in response to solar wind transient disturbances.

With regard to the need for certain conditions to make solar wind transients 'geoeffective' for producing TCVs, we would like to refer to a recent statistical investigation of solar wind pressure changes and the corresponding high-latitude impulsive responses by Sitar et al. (1996). They could not reveal a typical ground magnetic signature in response to transient dynamic pressure changes, but obtained a large variety of different kinds. The conditions to produce TCVs are obviously more complex than simple magnetospheric models predict for localised pressure changes.

Another feature that emerges from Fig. 2 is the tendency of TCV clusters to recur after 13 or 27 days. This feature implies that their occurrence is in some way linked to the solar rotation. To check this relation we compared our list of events with the distribution of the IMF sector structure shown in Fig. 1 of Crooker and McAllister (1997). We find that 9 events occur in the toward sector, 6 in the away sector and 2 during undetermined times. In contrast to the mentioned IMP8 observations which suggest a clear preference of the

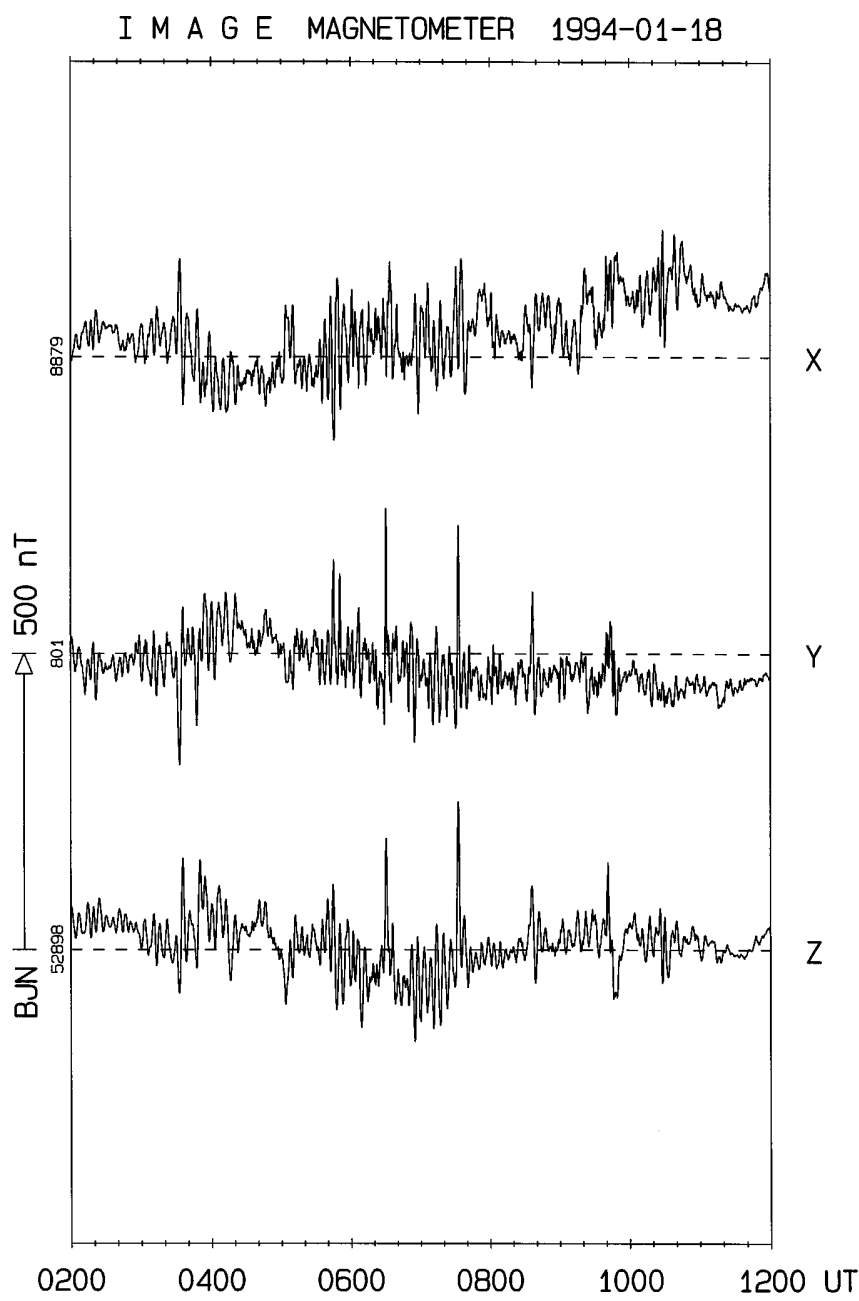

Fig. 8. Example for a day with multiple occurrences of TCVs. Solitary spikes in the $\mathrm{Y}$ component (positive or negative) are indications for TCVs in this pre-noon time sector

toward sector, these more complete data show that TCVs occur within statistical uncertainties equally often in both sectors. The apparent bias of the IMP- 8 data is caused by a beating between the many systematic data gaps over the 12-day orbit and the recurrence period of TCVs. In this light also the preference for a negative IMF $B_{Y}$, emerging from Table 3 , has to be reconsidered. In a toward sector a negative $B_{Y}$ is required to obtain the common IMF spiral geometry. Knowing that an almost equal number of events occurred in the away sector we have no longer observational evidence that negative IMF $B_{Y}$ is favourable for the formation of large-amplitude TCVs, as might have been implied by Table 3.

The 27-day recurrence plot of Dst index and sector structure (Fig. 1 of Crooker and McAllister, 1997) allow us to determine the phasing between the solar rotation and the events. When marking the dates of the events in the various panels we find clear evidence that our TCVs occur in the middle of the IMF sectors, not at the boundaries where the storms tend to start. During the six months of our survey there were two dominating sectors in the IMF. The toward sector lasting, on 
average, 15 days and the away lasting 12 days. From the superposition of Dst indices we deduced that large TCVs tend to occur 5 to 7 days after an onset of a storm. This finding fits quite nicley their appearance half way between the boundaries of the IMF sectors.

From this study we are not able to finally decide whether the clustering of TCVs during a certain phase of a recurrent storm pattern is controlled by the conditions in the magnetosphere or whether there are preferences for transient events in the solar wind half way between the sector boundaries. It requires more detailed observations in both regions to answer this question.

\section{Summary}

We have performed a statistical study of large-amplitude TCVs. The purpose of this study is to reveal and analyse any dependencies between TCVs and magnetospheric activity indices. The results of our statistics and superposed epoch analysis can be summarised as follows:

1. Large-amplitude TCVs occur in a narrow (2 hours) time sector centred at 10 MLT. All events travel westward.

2. On average the occurrence of large TCVs coincides with the location of the day-side convection reversal boundary of the two-cell pattern.

3. TCVs form during a quiet interval embedded in a moderately disturbed $\left(K p \sim 3^{+}\right)$period.

4. The observation of a positive IMF $B_{Z}$ and/or an increasing Dst value suggests that TCVs can develop large amplitudes only in a closed magnetosphere.

5. TCVs tend to occur in clusters distributed over a few days. These clusters repeat once or twice every 27 days. 6 . Within a magnetic storm cycle TCVs tend to occur some 5 days after the commencement.

7. At the same time there is also a clear tendency for TCVs to show up in the middle of IMF sector structures.

As a conclusion we like to speculate that TCVs require the magnetosphere to be in a certain state which allows them to grow to large amplitudes. These conditions could be distinctive but stable plasma boundaries which support the conversion of transient compressional perturbation at the magnetopause into field-aligned current filaments which subsequently drive ionospheric convection vortices.

We are aware of the limited statistical significance of our study caused both by the patchy solar wind data coverage and the limitation to a single magnetometer chain. During later years (e.g. 1995-1996) the situation has much improved in both respects and we propose to repeat a superposed epoch analysis taking advantage of the more complete data coverage. Our findings should be regarded as first results of a new approach for studying TCVs.

Acknowledgements. The motivation for this superposed epoch analysis of TCV events came from George Siscoe during the Geospace Environmental Modeling (GEM) Workshop 1996. The study has benefited greatly from discussions within this community. The IMAGE magnetometer network is a joint project of seven research groups in five European countries. We thank the funding agencies in the different countries for supporting this research facility.

Topical Editor K.-H. Glaßmeier thanks T. Moretto and R. Clauer for their help in evaluating this paper.

\section{References}

Baker, K. B., and S. Wing, A new magnetic coordinate system for conjugate studies of high latitudes, J. Geophys. Res., 94, 91399143, 1989.

Clauer, C. R., R. L. McPherron, and C. Searls, Solar wind control of low-latitude asymmetric magnetic disturbance field, J. Geophys. Res., 88, 2123-2130, 1983.

Crooker, N. U., and A. H. McAllister, Transients associated with recurrent storms, J. Geophys. Res., 102, 14,041-14,047, 1997.

Glassmeier, K.-H., Traveling magnetospheric convection twinvortices: observations and theory, Annales Geophysicae, 10, 547-565, 1992.

Glassmeier, K.-H., M. Hönisch, and J. Untiedt, Ground-based and satellite observations of travelling magnetospheric convection twin-vortices, J. Geophys. Res., 94, 2520-2528, 1989.

Goertz, C. K., E. Nielsen, A. Korth, K.-H. Glassmeier, C. Haldoupis, P. Hoeg, and D. Hayward, Observations of a possible ground signature of flux transfer events, J. Geophys. Res., 90, 40694078, 1985.

Friis-Christensen, E., M. A. McHenry, C. R. Clauer, and S. Vennestr $\varnothing \mathbf{m}$, Ionospheric traveling convection observed near the polar cleft: a triggered response to sudden changes in the solar wind, Geophys. Res. Lett., 15, 253-256, 1988.

Iyemori, T., T. Araki, T. Kamei, M. Takeda, Mid-latitude geomagnetic indices ASY and SYM (provisional), 4/5, Data Analysis Center for Geomag. and Space Magnetism, Kyoto University, Kyoto, 1994.

Kleymenova, N. G., O. V. Bolshakova, V. A. Troitskaya, and E. Friis-Christensen. Two forms of long period geomagnetic pulsations near the equatorial border of the dayside polar cusp, Geomagn. Aeron., 25, 139, 1985.

Lanzerotti, L. J., L. C. Lee, C. G. Maclennan, A. Wolfe, and L. V. Medford, Possible evidence of flux transfer events in the polar ionosphere, Geophys. Res. Lett., 13, 1089-1092, 1986.

Lanzerotti, L. J, R. D. Hunsucker, D. Rise, and L. Lee, Ionosphere and ground-based response to field-aligned currents near the magnetospheric cusp regions. J. Geophys. Res., 92, 7739-7743, 1987.

Lee, L. C. Magnetic flux transfer at the Earth's magnetopause, in Solar Wind-Magnetosphere Coupling, Eds Y. Kamide and J. A. Slavin, Terra, Tokyo, 297, 1986.

Lühr, H., The IMAGE magnetometer network, in STEP International, 4, USSCO; Oct. 1994.

Lühr, H. and W. Blawert, Ground signatures of travelling convection vortices, in Solar Wind Sources of Magnetospheric ULF Waves, Eds. M. J. Engebretsen, K. Takahashi and M. Scholer, AGU Geophys. Monograph 81, 231-251, 1994.

Lühr, H., W. Blawert, and H. Todd, The ionospheric plasma flow and current patterns of travelling convection vortices: a case study, J. Atmos. Terr. Phys., 55, 1717-1727, 1993

Lühr, H., M. Lockwood, P. E. Sandholt, T. L. Hansen, and T. Moretto, Multi-instrument ground-based observations of a travelling convection vortices event, Ann. Geophys. 14, 162181, 1996

Lühr, H., A. Aylward, S. C. Buchert, T. Holmboe, A. Pajunpää, K. Pajunpää, and S. M. Zalewski, Westward moving dynamic substorm features observed with the IMAGE magnetometer network and other ground-based instruments, Annales Geophysicae (in press) 1998.

Moretto, T., E. Friis-Christensen, H. Lühr, and E. Zesta, Global perspective of ionospheric traveling convection vortices: case 
studies of two Geospace Environmental Modeling events, J. Geophys. Res., 102, 11597-11610, 1997.

Ruohoniemi, J. M., and R. A. Greenwald, Statistical patterns of high-latitude convection obtained from Goose Bay HF radar observations, J. Geophys. Res., 101, 21743-21763, 1996.

Saunders, M. A., C. T. Russell, and N. Sckopke, Flux transfer events: scale size and interior structure, Geophys. Res. Lett., 11, 131-134, 1984.

Sibeck, D. G., A model for the transient magnetospheric response to sudden solar wind dynamic pressure variations, J. Geophys. Res., 95, 3755-3771, 1990.
Sitar, R. J., C. R. Clauer, and E. Friis-Christensen, High-latitude ground-based response to sudden changes in solar wind dynamic pressure, J. Geophys. Res., 101, 27001-27013, 1996.

Southwood, D. J., The ionospheric signature of flux transfer events, J. Geophys. Res., 92, 3207-3213, 1987.

Vo, H. B., J. S. Murphree, R. D. Elphinstone, R. C. Elphic, E. FriisChristensen, H. Lühr, and D. G. Sibeck, Multipoint observations of a dayside transient event, J. Geophys. Res., 99, 13409-13423, 1994. 